\title{
Location-Based Memory Fences
}

\author{
Edya Ladan-Mozes I-Ting Angelina Lee \\ MIT CSAIL \\ 32 Vassar Street, Cambridge, MA 02139 \\ \{edya, angelee\}@csail.mit.edu
}

\author{
Dmitry Vyukov* \\ OOO Google \\ 7 Balchug Street, Moscow, 115035, Russia \\ dvyukov@google.com
}

\begin{abstract}
Traditional memory fences are program-counter (PC) based. That is, a memory fence enforces a serialization point in the program instruction stream - it ensures that all memory references before the fence in the program order have taken effect before the execution continues onto instructions after the fence. Such PC-based memory fences always cause the processor to stall, even when the synchronization is unnecessary during a particular execution. We propose the concept of location-based memory fences, which aim to reduce the cost of synchronization due to the latency of memory fence execution in parallel algorithms.

Unlike a PC-based memory fence, a location-based memory fence serializes the instruction stream of the executing thread $T_{1}$ only when a different thread $T_{2}$ attempts to read the memory location which is guarded by the location-based memory fence. In this work, we describe a hardware mechanism for location-based memory fences, prove its correctness, and evaluate its potential performance benefit. Our experimental results are based on a software simulation of the proposed location-based memory fence, which incurs higher overhead than the proposed hardware mechanism would. Even though applications using the software prototype implementation do not scale as well compared to the traditional memmory fences due to the software overhead, our experiments show that applications can benefit from using location-based memory fences. These results suggest that a hardware support for locationbased memory fences is worth considering.
\end{abstract}

Categories and Subject Descriptors: C.1.m [Processor Architectures]: Miscellaneous; D.1.3 [Programming Techniques]: Concurrent Programming-Parallel programming

General Terms: Design, Performance, Theory

Keywords: location-based memory fences, memory fences, asymmetric synchronization, the Dekker duality, the Dekker protocol, biased locks

\footnotetext{
*This work was conducted by the author outside of Google.

This research was supported in part by the National Science Foundation under Grant CNS-1017058 and in part by the Angstrom Project funded by the Defense Advanced Research Projects Agency UHPC program under Agreement Number HR0011-10-9-0009.
}

Permission to make digital or hard copies of all or part of this work for personal or classroom use is granted without fee provided that copies are not made or distributed for profit or commercial advantage and that copies bear this notice and the full citation on the first page. To copy otherwise, to republish, to post on servers or to redistribute to lists, requires prior specific permission and/or a fee.

SPAA'11, June 4-6, 2011, San Jose, California, USA.

Copyright 2011 ACM 978-1-4503-0743-7/11/06 ...\$10.00.

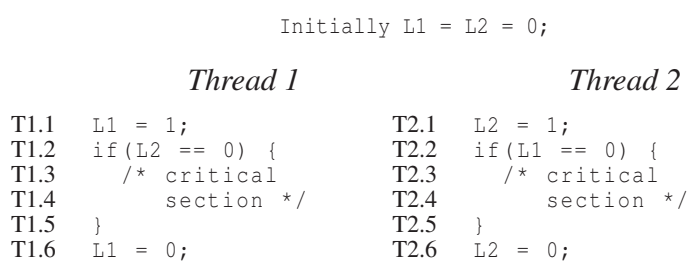

Figure 1: A simplified version of the Dekker protocol (omitting the mechanism to allow the threads to take turns), assuming sequential consistency.

\section{INTRODUCTION}

On many modern multicore architectures, threads ${ }^{1}$ typically communicate and synchronize via shared memory. Classic synchronization algorithms such as Dekker [10], Dijkstra [9], Lamport (Bakery) [18], and Peterson [22] use simple load-store operations on shared variables to achieve mutual exclusion among threads. All these algorithms employ an idiom, referred as the Dekker duality [6], in which every thread writes to a shared variable to indicate its intent to enter the critical section and reads the other's variable to coordinate access to the critical section.

Crucially, the correctness of such idiom rely on that the memory model exhibits sequential consistency (SC) [19], where all processors observe the same sequence of memory accesses, and within this sequence, the accesses made by each processor appear in its program order. While the SC memory model is the most intuitive to the programmer, existing systems typically implement weaker memory models that relax the memory ordering to achieve higher performance. The reordering does not affect the correctness of software execution for the most part, but in some cases, such as in the Dekker duality, it is important that the execution follows the program order, and the processors observe the relevant accesses in the same relative order.

Consider the following code segment shown in Figure 1, which is a simplified version of the Dekker protocol $[10]^{2}$ using the idiom to synchronize access to the critical section among two threads. If the read in line T1.2 gets reordered with the write in line T1.1 (and similarly for Thread 2), or if Thread 1 and Thread 2 observe different order of when the writes (lines T1.1 and T2.1) occur, an

\footnotetext{
${ }^{1}$ Throughout the paper, we use the terms thread and processor interchangeably. In particular, we use thread in the context of describing an algorithm and processor in the context of describing hardware features.

${ }^{2}$ This simplified version is vulnerable to livelock, where both threads simultaneous try to enter the critical section - each thread sets its own flag, reads the other thread's flag, retreats, and retries. Without some way of breaking the tie, the two threads can repeatedly conflict with each other and retry perpetually. The full version is augmented with mechanism to allow the threads to take turns and thus guarantees progress. For the sake of clarity, we present the simplified version here.
} 
incorrect execution may result, causing the two threads to enter the critical section concurrently.

To ensure a correct execution in such cases, these architectures provide serializing instructions and memory fences to force a specific memory ordering when necessary. Thus, a correct implementation of the Dekker protocol for such systems would require a pair of memory fences between the write and the read (between lines T1.1 and T1.2, and lines T2.1 and T2.2 in Figure 1), ensuring that the write becomes visible to all processors before the read is executed. Memory fences are costly, however, taking many more cycles to complete than regular reads and writes. Furthermore, a memory fence incurs overhead on the program execution even when the program is executed serially, or when the synchronization is unnecessary. For instance, the overhead incurred by the fence is unnecessary if only one thread intends to enter the critical section, and its write eventually becomes visible before another thread tries to enter the critical section.

Traditional implementation of memory fences is programcounter (PC) based. One cannot avoid the overhead incurred by a PC-based memory fence: upon execution the processor must stall, waiting for all outstanding writes before the fence in the instruction stream to become globally visible. The stalling is unnecessary, however, when no other threads are performing a read on these updated memory locations. In this work, we propose a locationbased memory fence, which causes the executing thread to "serialize" only when another thread tries to access the memory location associated with the memory fence. Location-based memory fences aim to reduce the latency in program execution incurred by memory fences. Unlike a PC-based memory fence, a location-based memory fence is conditional and remotely enforced - whether the executing thread serializes the memory accesses or not depends on whether there exists another thread that attempts to access the memory location associated with the memory fence.

Applications that employ the Dekker duality can benefit from location-based memory fences. While the Dekker duality seems to apply only to applications that synchronize between two threads, the idiom is commonly used to optimize applications involving multiple threads that exhibit asymmetric synchronization patterns, where one thread, the primary thread, enters a particular critical section much more frequently than the other threads running in the same process, referred as the secondary threads. Such applications typically employ an augmented version of the Dekker protocol: the secondary threads first compete for the right to synchronize with the primary thread (by grabbing an ordinary lock); once obtaining the right, the winning secondary thread then synchronizes with the primary thread using the Dekker protocol. The augmented Dekker protocol intends to speedup the execution path of the primary thread, even at the expanse of the secondary threads; therefore, it is particularly desirable to optimize away the overhead of memory fences on the primary thread's execution path, when the application executes serially or when there is no contention.

Many examples of such applications exist. For example, Java Monitors are implemented with biased locking [7, 16, 21], which uses an augmented version of the Dekker protocol to coordinate between the bias-holding thread (primary) and a revoker thread (secondary). Java Virtual Machine (JVM) employs the Dekker duality to coordinate between mutator threads (primary) executing outside of JVM (via Java Native Interface) and the garbage collector (secondary) [7]. In a runtime scheduler that employs a workstealing algorithm $[2-5,11,12,17]$, the "victim" (primary) and a given "thief" (secondary) coordinate the steal using an augmented Dekker-like protocol. Finally, in network package processing applications, each processing thread (primary) maintains its own data structures for its group of source addresses, but occasionally, a thread (secondary) might need to update data structures maintained by a different thread [23].

Such applications motivate our study of location-based memory fences. In these applications, we would like to tune the algorithm so that the primary thread operates on the fast path and avoid the overhead of memory fences when possible; only when a secondary thread attempts to enter the critical section, the secondary thread takes measures to ensure that the primary thread serializes, thereby achieving synchronization.

To evaluate the feasibility of location-based memory fences, we use a software prototype to simulate the effect of location-based memory fences and evaluate two applications with the software prototype. While the software implementation incurs higher overhead than the proposed hardware mechanism would, our experiments show that applications still benefit from the software implementation. These results suggest that a hardware support for location-based memory fences is worth considering.

The rest of the paper is organized as follows. Section 2 gives a abbreviated background on why reordering occurs in architectures that support a weaker memory model. Section 3 presents the proposed hardware mechanism for location-based memory fences. Section 4 formally defines the specification of location-based memory fences, proves that the proposed hardware mechanism implements the specification, and lastly shows that the Dekker protocol using location-based memory fences provides mutual exclusion. Section 5 evaluates the feasibility of location-based memory fences using a software prototype implementation with two applications. Section 6 gives a brief overview on related work. Finally, Section 7 draws concluding remarks.

\section{STORE BUFFERS AND MEMORY ACCESSES REORDERING}

In this section, we briefly review features of modern architecture design, which are necessary for our proposed hardware mechanism for location-based memory fences. In particular, throughout the rest of the paper, we assume that the target architecture implements either the Total-Store-Order (TSO) model (implemented by SPARC-V9 [24]) or the Process-Ordering (PO) model (implemented by Intel 64, IA-32 [15], and AMD64 architectures [1]). We also describe how memory reordering can occur, i.e., how the observable order in which memory locations are accessed can differ from the program order. Memory reordering can be introduced either by the compiler or the underlying hardware. Compiler fences that prevent the compiler from reordering have relatively small overhead, whereas the memory fences that prevent the reordering at the hardware level are much more costly. In this section, we focus on the reordering at the hardware level.

The target architecture we are considering supports out-of-order execution, but "commits" executed instructions in order. While the underlying hardware can freely reorder instructions, the result of committed instructions must still obey rules defined by the memory model implemented by the hardware. Specifically, the TSO and PO models conform to the following ordering principles for regular reads and writes issued by a given processor: ${ }^{3}$

1. Reads are not reordered with other reads;

2. Writes are not reordered with older reads;

\footnotetext{
${ }^{3}$ There are more ordering principles when one considers the interleaving of memory accesses issued by multiple processors and when one accounts for serializing instructions and memory fences; for the purpose of explaining the hardware mechanism, we only include a relevant subset. We refer interested readers to $[1,15,24]$ for full details.
} 


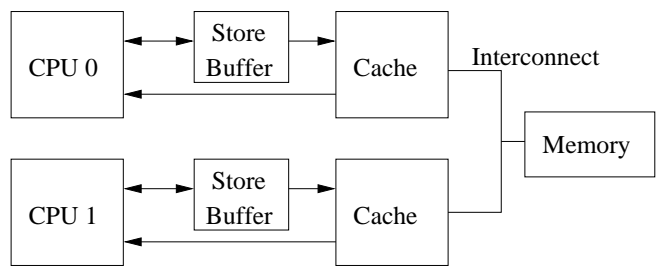

Figure 2: A simplified illustration of the relationship between the CPUs, the store buffers, and the memory hierarchy. Each CPU is connected with its own private cache. In addition, a store buffer is placed between the CPU and the cache, so that a write issued by the CPU is first stored in the store buffer and flushed out to the cache at later time. A read may be served by the cache, or by the store buffer if the store buffer contains a write to the same target address as the read.

3. Writes are not reordered with other writes; and

4. Reads may be reordered with older writes if they have different target locations (but they are not reordered if they have the same target location).

Principle 4 violates the Dekker duality, because it allows the read in line T1.2 of Figure 1 to appear to Thread 2 as if it has occurred before line T1.1, even though it appears as executed in order for Thread 1. The reason behind Principle 4 is to allow a typical optimization that modern architectures implement — writes performed by the processing unit are queued up in a private store buffer, which is a first-in-first-out (FIFO) queue instead of being written out to the memory hierarchy.

Figure 2 is a simplified illustration of the relationship between the processors (CPUs), the store buffers, and the memory hierarchy. Though not explicitly shown in Figure 2, the memory hierarchy in modern architectures typically consists of several levels of private and shared caches and the main memory. The further away the memory hierarchy is from the processor, the higher the latency it incurs. The use of a store buffer improves the performance of the program, because writing to a store buffer avoids the latency incurred by writing out to the cache. A write in the store buffer is only visible to the executing processor but not to other processors, however. Thus, from other processors' perspective, it appears as if the read has taken place before the older write (in program order). On the contrary, assuming that a cache coherence protocol is employed, a write becomes globally visible once its written to the cache, since the coherence protocol mandates accesses to data and enforces sequentially consistent view of the accessed data among the caches of all processors. As required by the proposed hardware mechanism for location-based memory fences, we assume that the target architecture employs the MESI cache coherence protocol [15], although the mechanism can be adapted to other variants such as MSI [13] and MOESI [1].

Now we define more precisely what we mean by committing the executed instructions in order. A read instruction is considered to be committed once the data is available (in at least Shared state) in the processor's private cache. A read may be speculatively executed out of order, but it must be committed in order. That is, the processor may perform a speculative read and fetch the cache line early, but if the cache line gets invalidated between the speculative read and when the read should commit in program order, the processor must reissue the read and fetch the cache line again. Once a read is committed successfully, the read value can be used in subsequent instructions.

A write instruction, on the other hand, has two phases: "committed" and "completed." A write is considered to be committed once it is written to the store buffer, although its effect is not yet visible by other processors. A write is considered to be completed, when it is flushed from the store buffer and written to the processor's cache. Once a write is completed, its effect becomes globally visible, since the cache coherence protocol ensures that all processors have a consistent view (the processor must gain Exclusive state on the flushed location before it update the value in the cache).

Since the guarantee is only that instructions must be committed in order, once the write is committed, the processor is free to continue executing subsequent instructions. A subsequent read instruction (with a different target address) may freely commit, even though an older write may still be in the store buffer. Thus, the resulting behavior observable by the other processors is that the read appears to have taken place before the older write.

The executing processor does not observe this reordering and always sees its own write, however, since the hardware employs store-buffer forwarding, so that a read with a target address that appears in the store buffer is serviced by the store buffer instead of by the cache. The store-buffer forwarding also enforces the ordering principle that a read is not reordered with an older write if they have the same target address. Furthermore, due to store-buffer forwarding, when two writes from two processors, say $P_{1}$ and $P_{2}$, interleave, the write ordering observed by $P_{1}$ may differ from the write ordering observed by $P_{2}$, because each processor always sees its own write as soon as it commits, but not the write performed by the other processor until the write reaches the cache. ${ }^{4}$

Whenever the system bus is available, the store buffer flushes the oldest entry to memory, so that each write becomes complete in FIFO order, ensuring that a write is never reordered with other writes (Principle 3). In the event that a context switch, an interrupt, or a serializing instruction (e.g., a memory fence) is encountered, the entire store buffer is drained as well, stalling the processor until all writes in the store buffer become globally visible.

\section{LOCATION-BASED MEMORY FENCES}

In this section, we describe location-based memory fences, or l-mfence in detail, including its informal specification, usage, and a proposed hardware implementation. The formal specification, as well as a correctness proof, is presented in Section 4. The proposed hardware mechanism that implements the l-mfence assumes an underlying architecture as described in Section 2.

\section{Informal Specification and Usage of l-mfence}

To describe the informal specification of 1 -mfence, we need to digress a bit and first describe the specification of an ordinary memory fence, or mfence. In Section 4 we give a formal definition of these ordering before we formally define the specification of the l-mfence.

The mfence instruction can be used to prevent other processors from observing reordering of the executing processor's instruction stream, at the point of a mfence execution - mfence simply forces the processor to stall until its store buffer is drained, flushing all its entries out to the cache in FIFO order. We say that the executing processor "serializes" its instruction stream at the point of mfence, meaning that the executing processor completes all the memory accesses prior to mfence, before executing instructions after mfence.

An l-mfence, unlike an ordinary memory fence, executes a memory fence "on demand." It takes in two inputs: a location $l$ guarded by the fence and a value $v$ to store in $l$ (see Figure 3(a)), and it serializes the instruction stream of the executing processor only when another processor attempts to access the guarded memory location.

\footnotetext{
${ }^{4}$ While $P_{1}$ and $P_{2}$ may observe different orders, the other processors in the system will observe a consistent ordering of the two writes.
} 
Primary Thread

\begin{tabular}{|c|c|}
\hline $\mathrm{K} 1$ & 1 -mfence $(\& L 1,1)$ \\
\hline K2 & if $(\mathrm{L} 2==0) \quad i$ \\
\hline K3 & /* critical \\
\hline K4 & section $* /$ \\
\hline K5 & \\
\hline K6 & $\mathrm{L} 1=0 ;$ \\
\hline
\end{tabular}

\section{Secondary Thread}

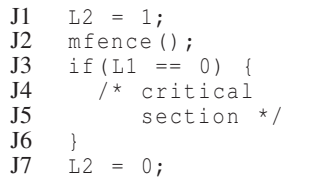

(a)

Instruction translation for l-mfence $(L 1,1)$ (line K1 in Thread 1)

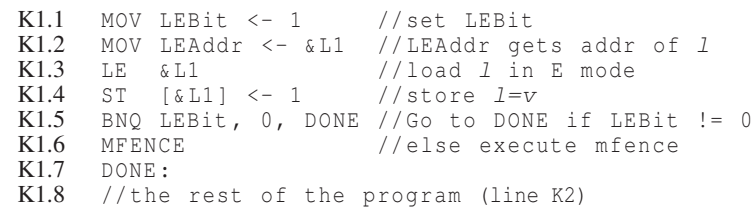

(b)

Figure 3: (a) The asymmetric Dekker protocol using location-based memory fences. The code for the primary thread is shown in lines K1-K6, and the code the secondary thread is shown in lines J1-J7. (b) The instructions generated for 1 -mfence shown in line $\mathrm{K} 1$ in the code for the primary thread in (a).

The serialization of $P$ 's instruction stream enforces a relative ordering between the store $S$ associated with the execution of 1 -mfence and the other accesses performed by $P$. The ordering between $S$ and an access $A$ are observed consistently across processors, including the processor executed the 1 -mfence and $A$. That is, from $P$ 's perspective, if $P$ executed $S$ before (after) $A$, all processors observe that $S$ "happened" before (after) $A$. The serialization does not enforce any relative ordering between accesses that happen before (after) $S$, however, meaning that the l-mfence ensures that all processors (including $P$ ) consistently observe that $A_{1}$ and $A_{2}$ happened before (after) $S$, but they may not have a consistent view of the relative ordering between $A_{1}$ and $A_{2}$. The relative ordering between these accesses is still defined by the TSO / PO memory model.

The use of 1 -mfence is very similar to the use of mfence, except that the 1 -mfence is associated with a specific store. A in mfence, when l-mfence is used in the program, an implicit compiler fence should be inserted in place to prevent reordering at the compiler level. Threads synchronizing via $l$-mfence need to coordinate with each other and be careful as to where to place the l-mfence and which memory location to guard / read after. Since a l-mfence does not guarantee atomic read-modify-write operation, its correct usage typically involves single writer only. Note that 1 -mfence prevents other processors from observing the reordering of the executing processor's instruction stream, but it does not prevent the executing processor from observing reordering of other processors' instruction streams. Therefore, correct usages of $l$-mfence typically consist of a pair of memory fences. For instance, to ensure correct execution in the case of the Dekker protocol, it is crucial that both processors insert memory fences between the write and the read, to prevent the other processor from observing reordering. For 1 -mfence, the pairing can be with either another 1 -mfence or an ordinary mfence.

\section{Hardware Implementation of l-mfence}

Our proposed implementation of 1 -mfence requires a new hardware mechanism, called load-exclusive / store, or LE/ST . Conceptually, the LE/ST mechanism allows the processor to setup a "link" to keep track of the status of the store associated with the l-mfence (i.e., whether the store to guarded location is committed or completed as defined in Section 2). It also allows the processor to coordinate with the cache controller to monitor attempts to access the guarded location. Another processor's attempt to access the guarded location causes the processor to clear the link and triggers actions necessary to serialize the instruction stream. On the other hand, if the store becomes complete before another processor attempts to access the guarded location, the processor clears the link and thus stops guarding the location.

$\mathrm{LE} / \mathrm{ST}$ requires one new instruction and two additional hardware registers. The new instruction, LE, takes one operand - the location of the variable to load, and obtains Exclusive state on that location. Therefore, once LE is committed, the processor has the location in its cache in Exclusive state, and no other processors have a valid copy of the location in their cache. Since LE is very similar to a regular load, except the requirement for Exclusive state on the location, it can be easily implemented by modern architectures using the MESI coherency protocol. The two additional hardware registers are LEBit and LEAddr, both readable and writable by the processor, and readable by the cache controller. The processor must update these register to enable the link and guard the memory location specified by the $1-$ mfence. First we describe how the processor updates these registers to setup the link, and then we describe how the processor and the cache controller coordinate with each other to guard the memory location.

Figure 3(b) presents the assembly-like translation for the l-mfence $(1, \mathrm{v})$ where $l==L 1$ and $v==1 .^{5}$ Initially, LEBit and LEAddr are cleared. As part of the 1 -mfence $(\& L 1,1)$, the processor starts to create the link to the guarded location by setting the LEBit with 1 and LEAddr with $L 1$ (lines K1.1 and K1.2 in Figure 3(b)). Next, the LE instruction in line K1.3 loads $L 1$ into the cache in Exclusive state, so that no other processor holds a copy of L1 in its cache. At this point we say that the link is set. The ST instruction in line K1.4 stores the value 1 to L1, committing it into the store buffer. If for any reason the link is broken, implied by the zero value in LEBit (line K1.5), the processor executes a MFENCE (line K1.6). The MFENCE causes the processor to serialize its execution - it flushes the store buffer, and by that it completes the store of the guarded location, making it globally observable by other processors. If the link is not broken when the ST in line K1.4 commits, the processor may continue without flushing the store buffer.

We now explain how the cache controller interacts with the processor to guard the location stored in LEAddr. Whenever both LEBit and LEAddr are set, the cache controller listens to cache coherency traffic, and notifies the processor if any request requires the controller to either (1) downgrade the cache line corresponding to the memory location stored in LEAddr from Exclusive state; or (2) evicts the cache line. The cache controller then waits for the processor's response before it takes any actions regarding the guarded location, since these events break the link to the guarded location.

When the processor receives the notification from the cache controller, it clears the LEBit and LEAddr, flushes the store buffer, and replies to the cache controller. At the time the processor replies the cache controller, the most up-to-date value of the guarded location is already in the cache. When the cache controller gets the processor's reply, it resumes the actions it needs to take regarding the guarded location. By clearing the LEBit, the processor remembers that the link to the guarded location is broken. In the event that the link is broken before ST (line K1.4) was committed, the code for

\footnotetext{
${ }^{5}$ The code shown is not strictly assembly. First, we are not using a particular instruction set. Second, for the sake of clarity, we choose to use the store instruction (line K1.4) instead of using the regular move instructions to specify instructions that write to memory (i.e., non registers).
} 
l-mfence takes the branch that executes an MFENCE, causing the store buffer to flush (line K1.5) after the store commits.

The link remains set for as long as the primary processor still has the cache line, until the corresponding store to the guarded location is complete. When the corresponding store in the store buffer is flushed, possibly due to other internal reasons (for instance, the store is naturally flushed as the oldest entry in the buffer, the buffer is full, or a context switch occurs), upon completing the store, the processor also clears LEBit and LEAddr. The guarded location can still remain in the cache in Exclusive (or Modify) state if there is no request to evict or downgrade it.

In the context of the Dekker protocol, since LE ensures that the primary processor has the cache line for $\mathrm{L} 1$ in Exclusive state before the ST in line K1.4, its cache controller must receive a downgrade request from a secondary processor before the secondary processor can access L1. Furthermore, since the cache controller of the primary processor cannot respond to the downgrade request until the primary processor replies, the secondary processor will see the most up-to-date value of L1. Essentially, we piggyback on the cache coherence protocol to detect another processor's attempt to access the guarded location. We also rely on the coherency protocol to deliver the most up-to-date value to the other processor, since the store buffer is flushed before the cache controller replies to the secondary processor. It is necessary for the cache controller to notify the processor when it needs to evict the cache line, since the cache controller can no longer help guarding the memory location, if the given cache line is evicted.

The design of this hardware mechanism is intended to be lightweight and efficient. Since we assume only one pair of LEBit and LEAddr are allocated per processor, if a processor encounters a second LE/ST while the link from the first LE/ST is still in effect, the processor must flush the store buffer, clear LEBit and LEAddr, before it can proceed with the second LE/ST. unless the second LE/ST has the same target load address as the first LE/ST.

\section{Expected Overhead of the LE/ST Mechanism}

The LE/ST mechanism ensures that the primary processor, when running alone, will not execute any memory fences, and perform only regular stores; it still needs to perform LE regardless, but that should not incur much overhead - the hope is that the target cache line of LE stays in the primary processor's cache. Furthermore, the target cache line of $\mathrm{LE}$ only gets invalidated whenever the secondary processor attempts to enter the critical section. Assuming the secondary processor synchronizes infrequently, the target cache line stays in primary processor's cache between the secondary processor's synchronization attempts. Finally, the primary processor flushes the store buffer only when the secondary processor attempts to enter the critical section when the link is in effect, so the primary processor should perform regular store for the most part. On the other hand, the secondary processor could stall for a while when waiting for the primary processor to flush its store buffer, but the assumption is that the secondary processor can incur overhead if it improves the performance of the primary processor.

If the secondary processor synchronizes frequently, each use of l-mfence may result in one store buffer flush, which is comparable to a regular mfence. There is once case in which the /Fast processor will flush the buffer twice - if a downgrade request (due to a secondary processor attempts to access the guarded memory location) arrives at the primary processor between the commit of LE (line K1.3) and ST (line K1.4). The first flush is performed when the processor is notified, and the second flush is performed after the ST commits, via taking the branch (lines K1.5 and K1.6). During the first flush, the guarded location is not committed to the store buffer yet. We choose to flush the store buffer at this point, because it results a more intuitive specification for the l-mfence. The second flush, on the other hand, is essential to guarantee correctness. Without the second flush, one could construct a scenario in which the secondary processor enters the critical section twice, once before the primary processor commits its store to L1 and once after, wrongfully allowing both processors to execute in the critical section concurrently. Even though the processor flushes the store buffer twice, the second flush contains only on location, the guarded location.

Lastly, the cache controller needs to compare the incoming request from cache coherence traffic against the address stored in LEAddr when the LE/ST mechanism is in effect. We believe that this comparison can be done in parallel with other operations that the cache controller already performs to handle the request, and thus it should not incur additional performance penalty.

\section{FORMAL SPECIFICATION AND CORRECTNESS OF L-MFENCE}

In this section, we formally define the specification of 1 -mfence and prove that the hardware mechanism described in Section 3 implements the specification. ${ }^{6}$ Then, based on the specification of 1 -mfence, we prove that the asymmetric Dekker Protocol using l-mfence (as shown in Figure 3(a)) achieves mutual exclusion.

\section{Formal Specification of l-mfence}

To formally define the specification of 1 -mfence, we first define the serialization order for a given memory location.

DEFINITION 1. Given a memory location $l$, the serialization order of accesses to l performed by all processors is as follows.

1. A load L from location $l$ is serialized after a store $S$ of $v$ to $l$ if and only if $L$ observes $v$.

2. A store $S$ of $v$ to location l performed by a processor $P$ is serialized after a store $S^{\prime}$ of $v^{\prime}$ to $l$ if at the time of completion of $S$, had P executed a load, the load would have observed $v^{\prime}$ from $S^{\prime}$.

3. A load L from $l$ is serialized before a store $S$ of $v$ to $l$ if there exists a store $S^{\prime}$ to l such that $L$ is serialized after $S^{\prime}$, and $S$ is also serialized after $S^{\prime}$.

Note that the serialization order involving stores are defined by the time of completion, not commit. To complete a store of $v$ to $l$, the executing processor $P$ must gain Exclusive state on $l$, and thus it can be viewed as if the store was preceded by a load from $l$, since the value of $l$ exists in $P$ 's cache in Exclusive state. Furthermore, since the serialization order on a location $l$ is defined by the completion time of stores, all processors agree on a single serialization order.

Definition 1 defines the serialization order on a given memory location that is globally consistent. Program order, on the other hand, is defined for a given processor, which is the order of memory accesses occurred in a processor $P$ 's instruction stream from $P$ 's perspective. If we consider all memory accesses from every processor to every memory location, there exists a global visibility order on these accesses (a posteriori), where the visibility order is consistent with the serialization order for each memory location and the ordering priciples defined by the TSO / PO model relative to each processor's program order.

\footnotetext{
${ }^{6}$ The definitions we describe in this section in order to formally define the specification for 1 -mfence are similar to certain definitions described in [14], although we use different notations and terminology, and define only the terms we need.
} 
Given the visibility order of a particular execution, we say that a memory access $A_{1}$ happened before (after) another access $A_{2}$ if $A_{1}$ precedes (follows) $A_{2}$ in the visibility order, or $A_{1}<A_{2}$. From $P$ 's perspective, we say that a memory access $A_{1}$ occured before (after) another access $A_{2}$ if $A_{1}$ precedes (follows) $A_{2}$ in $P$ 's program order, or $A_{1} \ll A_{2}$.

Now we define the specification of 1 -mfence formally.

DEFINITION 2. Given a store $S$ associated with $I$-mfence executed by a processor $P$, and an access $A$ also performed by $P$, the $I-m f e n c e$ enforces that if $A \ll S$ then $A<S$, and vice versa, without breaking the TSO / PO ordering principles.

An $l$-mfence $(l, v)$ performed by processor $P$ executes a store $S$ of $v$ to $l$, and enforces a happened-before (after) relation between $S$ and any other access $A$ performed by $P$ that is consistent with $S$ and $A$ 's relative ordering in $P$ 's program order. That is, if access $A$ occurred before (after) 1 -mfence in $P$ 's instruction stream, $A$ appears to all processors that it has happened before (after) $S$ in the global visibility order.

\section{Correctness Proof of the LE/ST Mechanism}

We start by some definition and lemmas that will help us show that the LE/ST mechanism (which includes the code sequence shown in Figure 3(b)) implements the specification of 1 -mfence.

DEFINITION 3. Given the LE/ST mechanism and a particular instance of $I-m f e n c e(I, V)$, a link for the $I-m f e n c e$ is set if LEBit contains 1, LEAddr contain l, and the cache line for $l$ is in the executing processor's private cache in Exclusive or Modified state. If any of these conditions is not met, the link is clear.

LEMMA 1. Given a particular instance of $I-m f e n c e(I, V)$, if LEB it contains 1 when the store commits (line K1.4), the link must be set.

PROOF. By committing instructions shown in lines K1.1-K1.3, the executing processor set up the link. Since LEBit is set as the first instruction of the l-mfence execution, if the link was broken at any point before the commit of ST in line K1.4, the LE/ST mechanism clears LEBit as part of breaking the link. Once the link is broken, LEBit is never set again until the next instance of l-mfence.

LEMMA 2. The LE/ST mechanism maintains the ordering principles defined by the TSO / PO memory model.

PROOF. The LE/ST mechanism uses regular loads ${ }^{7}$, stores, and memory fences, which maintains the FIFO ordering in the store buffer and the fact that instructions are committed in order. Thus, the TSO / PO principles are maintained.

LEMMA 3. The LE/ST mechanism ensures that, before $P_{1}$ commits the next instruction following $I-m f e n c e(I, V)$, either the store $S$ to $l$ in line K1.4 is already complete, or any other access to $l$ from another processor $P_{2}$ must happen after $S$.

PROOF. There are two cases to consider - either the link is clear at the time when $S$ commits (Case 1), or the link is still set (Case 2).

Case 1: By Lemma 1, we know that if the link is clear, the LEBit must be 0 . Therefore, by the code for LE/ST mechanism (Figure 3(b)), the condition for the branch (line K1.5) is false, and thus

\footnotetext{
${ }^{7}$ As explained in Section 2, the LE instruction is very similar to a regular load and can be implemented using the existing architecture and cache coherency protocol.
}

$P_{1}$ must execute the MFENCE in line K1.6, causing $S$ to complete before the next instruction (line K2 in Figure 3(a)) commits.

Case 2: If the link is set, by Definition 3, we know that $P_{1}$ has $l$ in Exclusive / Modify state. Therefore, any processor $P_{2}$ will issue coherence traffic to $P_{1}$ before $P_{2}$ can commit a load from $l$ or complete a store to $l$ (a store must acquire Exclusive state on the location before it can complete). Since the link is set, $P_{1}$ 's cache controller will notify the processor when such request arrives. By the LE/ST mechanism upon notification, $P_{1}$ clears the link, flushes its store buffer to complete $S$, and replies to the cache controller. Only after that, the cache controller responds to $P_{2}$ 's request. Thus, $P_{2}$ 's access to $l$ happened after $S$.

THEOREM 4. The LE/ST mechanism implements the specification of I-mfence as defined in Definition 2.

PROOF. To show that the LE/ST mechanism implements the specification of 1 -mfence, we show that 1 -mfence enforces that if $A \ll S$ then $A<S$ and vice versa. By Lemma 2, we know that the LE/ST mechanism maintains the TSO / PO principles. Thus, the case where $A \ll S$ (for $A$ being either a load or a store) is trivially true. Similarly, the case where $S \ll A$ where $A$ is another store to a different location is also trivially true. Moreover, since the visibility order is always consistent with the serialization order to a given location, the case where $S \ll A$ where $A$ loads from or stores to the same target location as $S$, is also trivially true. Thus, the only case we need to analyze is $S \ll A$, where $A$ is a load with a different target location, and we show that $S<A$.

Let $P_{1}$ be the processor executing the 1 -mfence $\left(l_{1}, \mathrm{v}\right)$, and its program order dictates that the store $S$ to $l_{1}$ (associated with the l-mfence) happened before a load $A$ from location $l_{2}$. Assume for the purpose of contradiction, that some processor $P_{2}$ observes that $S$ happened after $A$. The only way that $P_{2}$ can observe such happenedafter relation is if $P_{2}$ performs some operations $B$ accessing $l_{2}$ and $C$ accessing $l_{1}$ in such way that $S$ is forced to happen after $A$. That is, based on the TSO / PO principles and the serialization order observed by $P_{2}$ during execution, $S$ cannot happen before $A$.

We consider possible candidates for $B$ and $C$. In order to enforce an ordering on $B$ and $C$ in visibility order, we cannot have $B$ being a store and $C$ being load, because the TSO / PO principles does not enforce that $B<C$ if $B \ll C$. We will not consider $B$ being a load, because we wish to enforce a visibility order between $A$ (also a load) and $B$ based on the serialization order of $l_{2}$, which is determined by stores completed on $l_{2}$. Involving an additional store on $l_{2}$ to force a serialization order between $A$ and $B$ is essentially the same effect as simply choosing $B$ as a store. Thus, we only consider the case in which both $B$ and $C$ are store operations.

With $B$ storing to $l_{2}$ and $C$ storing to $l_{1}$, we construct a scenario to obtain the visibility order $A<B<C<S$. Since $B \ll C$ in $P_{2}$ 's program order, we have $B<C$ as dictated by the TSO / PO model (Principle 3 in Section 2). We can obtain $A<B$ via the serialization order, since they both operate on memory location $l_{2}$ (assuming $B$ is serialized after $A$ ). Similarly, we can obtain $C<$ $S$ via the serialization order, since they both operate on memory location $l_{1}$.

Given this visibility order, we know that $A$ (a load) must commit before $B$ completes, otherwise $A$ would observe the value stored by $B$ and therefore serialize after $B$. Similarly, $C$ must complete before $S$ completes, otherwise $S$ would serialize before $C$. We also know that $B$ must complete before $C$ completes, by the TSO / PO principles. That means, $A$ must commit before $S$ completes. There are two cases to consider here.

Case 1: The link for the l-mfence that $S$ is associated with is clear when $A$ commits. By Lemma 3 , since $S$ must complete before 
the next instruction (following 1 -mfence) commits, we know that this visibility order cannot occur, and $S<A$.

Case 2: The link is set when $A$ commits. In this case, $S$ is committed but not yet complete when $A$ commits. Let's name the next immediate access to $l_{1}$ that completes as $D$ (possibly from any processor). By Lemma 3, $D$ must happen after $S$, i.e., $S<D$. If $D$ turns out be $C$, then $S<C$, and there is no reason why we cannot rearrange the visibility order to obtain $S<A<B<C$, since there is no ordering constraint that prevents $S$ from moving upward. If $D$ is not $C$, then $C$ must complete and happen after $D$, so we still have $S<C$. With the same reasoning, we can rearrange the visibility order to obtain $S<A<B<C$.

In both cases, we have $S<A$, which agrees with $P_{1}$ 's program order, $S \ll A$.

Theorem 4 proves that the LE/ST mechanism correctly implements the specification of 1 -mfence. In the following subsection, we prove that this specification is sufficient to guarantee mutual exclusion if it is used by the primary thread in the asymmetric Dekker protocol.

\section{Correctness Proof of the Asymmetric Dekker Algo- rithm using l-mfence}

We now prove that the $l$-mfence $(I, v)$ specification is sufficient for achieving mutual exclusion when it is used in the asymmetric Dekker protocol, such as shown in Figure 3(a). The proof is based on two lemmas, each shows that if one thread is running in its critical section, the other one is prevented from entering it. For brevity, we name the primary thread executing the l-mfence $T 1$ (lines K1-K6 in Figure 3(a)) and the secondary thread $T 2$ (lines J1-J7 in Figure 3(a)).

LEMMA 5. Assuming both $T 1$ and $T 2$ are concurrently attempting to enter the critical section. If $T 1$ reads that $L 2==0$ in line $K 2$ and is therefore entering the critical section, $T 2$ will not enter the critical section.

Proof. If $T 1$ reads that $L 2==0$ in line K2, we know that the load in line $\mathrm{K} 2$ must have committed before the store in line $\mathrm{J} 1$ completed. That is, the load in line $\mathrm{K} 2$ happened before the store in line $\mathrm{J} 1$. Since $T 2$ uses an mfence (line J2) between lines $\mathrm{J} 1$ and $\mathrm{J} 3$, the load in line $\mathrm{J} 3$ cannot execute until the store in line $\mathrm{J} 1$ completes. Thus, the load in line K2 must also have happened before the load in line J3. By the specification of 1 -mfence (Definition 2), since the store to $L 1$ associated with the 1 -mfence in line $\mathrm{K} 1$ must appear to happen before the load in line $\mathrm{K} 2$ to all processors, $T 2$ must observe that the store in line $\mathrm{K} 1$ happened before the load in line $\mathrm{K} 2$, which happened before the load in line J3. Therefore, when $T 2$ executes the load in line J3, it must observe the store performed in line $\mathrm{K} 1$, read $L 1==1$ (assuming $T 1$ has not left the critical section), and refrain from entering the critical section.

LEMMA 6. Assuming both $T 1$ and T2 are concurrently attempting to enter the critical section. If $T 2$ reads that $L 1==0$ in line J3 and is entering the critical section, T1 will not enter the critical section.

PROOF. If $T 2$ reads that $L 1==0$ in line $\mathrm{J} 3$, we know that the load in line J3 must have been serialized before the store that is associated with the 1 -mfence $(L 1,1)$. Thus, the load in line J3 happened before the the store for 1 -mfence $(\& L 1,1)$ in line K1. By the specification of 1 -mfence (Definition 2), T2 must observe that the store associated with line K1 happened before the load in line $\mathrm{K} 2$, so the load in line $\mathrm{J} 3$ must also have happened before the load in line K2. Since $T 2$ uses an mfence (line J2) between lines J1 and $\mathrm{J} 3$, the load in line $\mathrm{J} 3$ cannot execute until the store in line $\mathrm{J} 1$ completes, and thus the store in line $\mathrm{J} 1$ must also have happened before the load in line K2. Thus, when $T 1$ executes the load in line K2, $T 1$ must observe $T 2$ 's store to $L 2$ and read $L 2==1$ (assuming $T 2$ has not left the critical section), and refrain from entering the critical section.

THEOREM 7. The asymmetric Dekker protocol using I-mfence allows at most one thread to execute in the critical section at any given time.

PROOF. Follows form Lemmas 5 and 6.

As we have shown, the asymmetric Dekker protocol shown in Figure 3(a) guarantees mutual exclusion. Nonetheless, it requires additional tie-breaking code (similar to the original Dekker protocol) to avoid live lock situations in which both threads are kept outside their critical sections.

The asymmetric Dekker protocol is designed to optimize away the overhead incurred onto the primary thread at the expanse of additional overhead on the secondary thread, which is advantageous for applications that exhibit asymmetric synchronization patterns. Hence, we use an mfence in the secondary thread instead of 1 -mfence to avoid incurring additional overhead on the primary thread. If the secondary thread was using an l-mfence, the primary thread may need to wait for the secondary thread to flush its store buffer when it attempts to read L2 in line K2. Nevertheless, the secondary thread has the option of executing the mirrored code (using 1 -mfence $(\& L 2,1)$ in line $\mathrm{J} 2$ ), and the protocol still provides mutual exclusion in such case.

\section{EVALUATION}

To evaluate the feasibility of location-based memory fences, we have implemented a software prototype of location-based memory fences using signals. The idea of using signals to cause a thread to serialize has been proposed by Dice et al. in [6], but was not evaluated. We implemented the signal-based serialization method similar to what [6] proposed, and use it as a basis for evaluating the expected performance of the hardware mechanism for locationbased memory fences. We incorporate the software prototype of the fences into two applications. In this section, we briefly summarize the software prototype, the experimental setup, and the results of our evaluation.

\section{Software Prototype of l-mfence}

The software prototype must correctly capture two main effects. First, the primary thread must not reorder the write and the read at the compiler level. We achieve this simply by inserting a compiler fence at the appropriate place. Second, before the secondary thread attempts to read the primary thread's flag, it must cause the primary thread to serialize, and only proceed to read the flag when it knows that the primary thread has performed serialization. We achieve this via signals - a software signal generates an interrupt on the processor receiving the signal, and the processor flushes its store buffer before calling the signal handling routine. Thus, the secondary thread sends a signal to the primary thread and waits for an acknowledgment by spinning on a shared variable. ${ }^{8}$ Upon receiving the signal (which implicitly flushes the store buffer), the primary thread executes a user-defined signal handler, which sets the shared variable as an acknowledgment, thereby allowing the secondary thread to resume execution.

\footnotetext{
${ }^{8}$ This is assuming that the signaling succeeds; the signaling would fail if the primary thread has already terminated.
} 
This software prototype implementation incurs overhead that the proposed hardware mechanism would not. First, since the signal handler is user-defined, upon receiving the signal, the primary thread would need to cross between kernel and user modes four times, which incurs high overhead. The same overhead is incurred on the secondary thread as well, since the secondary thread must wait for the primary thread to acknowledge receiving the signal. Second, the secondary thread may observe some latency if the primary thread is de-scheduled from the processor. ${ }^{9}$ The proposed hardware mechanism would not observe these overheads.

On the other hand, in the software implementation, the fences incur virtually no overhead on the primary thread when there is no contention. This is not the case for the hardware mechanism, which incurs a small overhead on the primary thread. This overhead results from the registers setup, the branch to check LEBit, and the wait for Exclusive state on the guarded location. When there is no contention, however, most of this overhead is eliminated by the successful branch prediction and the fact that the location stays in the primary thread cache in Exclusive or Modified state, because no other thread is trying to access it. When there is contention, we speculate that the signal handling overhead associated with the software implementation are much higher compared to the hardware mechanism. Even though the software implementation does not faithfully simulate the overhead of the hardware mechanism, it nonetheless gives us some idea as to whether the hardware mechanism is worth investigating.

\section{Experimental Setup}

We incorporate the software prototype of the fences into two applications - the asymmetric Cilk-5 runtime system and an asymmetric multiple-readers single-writer lock.

For the first application, we have modified the open-source Cilk5 runtime system $[11]^{10}$ to incorporate the location-based memory fence into its Dekker-like protocol employed by the runtime work stealing scheduler, referred as the ACilk-5 runtime. In ACilk-5 runtime, when a thief (the secondary thread) needs to find more work to do, it engages in the asymmetric Dekker-like protocol with a given victim (the primary thread) in order to "steal" work from the victim's "deque." If there are more than two thieves attempt to steal from the same victim, the thieves must first compete for the right to engage the victim via a lock acquisition, so that only a single thief would engage in the Dekker protocol with the victim at any given moment. Assuming the application contains ample parallelism, a victim would access its own deque much more frequently than a thief, because steal occurs infrequently.

For the second application, we have designed an asymmetric multiple-readers single-writer lock, where the lock is biased towards the readers, henceforth referred as the $\boldsymbol{A} \boldsymbol{R} \boldsymbol{W}$ lock. From time to time, a reader (the primary thread) turns into a writer (the secondary thread), and attempts to acquire the ARW lock in write mode by engaging in the asymmetric Dekker protocol with each of the registered readers. Similarly to ACilk-5, if there are more than one writer at a given moment, the writers compete for permission to write by first acquiring a lock, and only the winning writer is allowed to engage in the Dekker protocol with the readers.

We ran all experiments on an AMD Opteron system with 4 quadcore $2 \mathrm{GHz}$ CPU's having a total of 8 GBytes of memory. Each core

\footnotetext{
${ }^{9}$ The correctness of the protocol is not affected by the primary thread being de-scheduled, because the de-scheduling constitutes a context switch, which requires the store buffer to drain before the de-scheduling.

${ }^{10}$ The open-source Cilk-5 system is available at http://supertech. csail.mit.edu/cilk/cilk-5.4.6.tar.gz.
}

\begin{tabular}{l|l|l} 
Application & Input & Description \\
\hline cholesky & $4000 / 40000$ & Cholesky factorization \\
cilksort & $10^{8}$ & Parallel merge sort \\
fft & $2^{26}$ & Fast Fourier transform \\
fib & 42 & Recursive Fibonacci \\
fibx & 280 & Alternate between fib(n-1) and fib(n-40) \\
heat & $2048 \times 500$ & Jacobi heat diffusion \\
knapsack & 32 & Recursive knapsack \\
lu & 4096 & LU-decomposition \\
matmul & 2048 & Matrix multiply \\
nqueens & 14 & Count ways to place $N$ queens \\
rectmul & 4096 & Rectangular matrix multiply \\
strassen & 4096 & Strassen matrix multiply
\end{tabular}

Figure 4: The 12 benchmark applications.

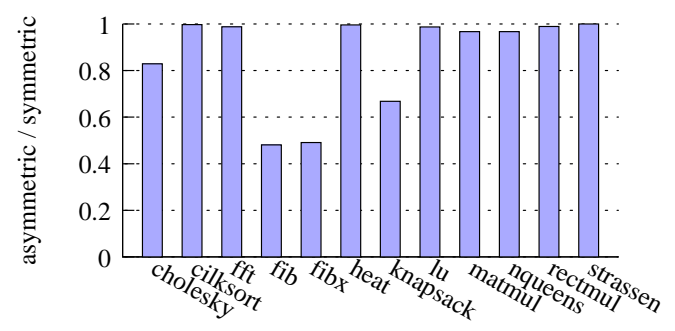

relative execution time on single processor

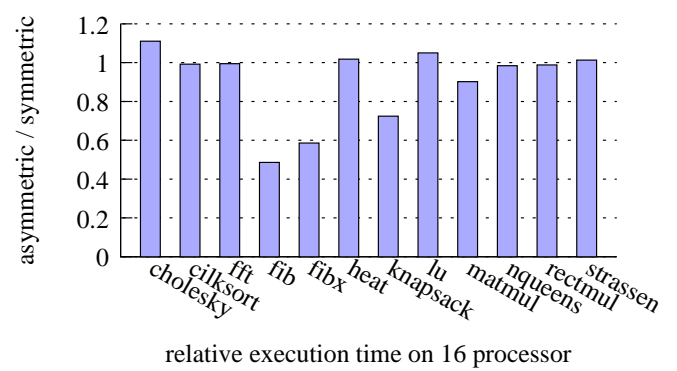

Figure 5: (a) The relative serial execution time of the ACilk-5 runtime system compared to the original Cilk- 5 runtime system for 12 Cilk benchmarks. (b) The relative execution time of the ACilk-5 runtime system compared to the original Cilk-5 runtime system for 12 Cilk benchmarks on 16 cores. A value below 1 means that the application runs faster on ACilk-5 than on Cilk-5; a value above 1 means the other way around. Each value is calculated by normalizing the execution time of the benchmarks on ACilk-5 with that on Cilk-5.

on a chip has a 64-KByte private L1-data-cache and a 512-KByte private L2-cache, but all cores on a chip share a 2-MByte L3-cache.

\section{Evaluation Using ACilk-5}

To evaluate the effect of location-based memory fences, we compare the execution time of applications running on ACilk-5 versus running on Cilk- 5 across 12 benchmarks. Figure 4 provides a brief description of each benchmark.

Figure 5(a) compares the performance of the benchmarks run on ACilk-5 and Cilk-5 when executed serially. Figure 5(b) shows a similar performance comparison when executed on 16 cores. For each measurement, we took the mean of 10 runs (with standard deviation of less than 3\%). A value below 1 means that the application runs faster on ACilk-5 than on Cilk-5. Not surprisingly, when executed serially, ACilk-5 runs faster, because the victim executes on the fast path with virtually no overhead from memory fences. The improvement that ACilk-5 exhibits over Cilk-5 when running a given benchmark is directly related to the number and the granularity of tasks that the benchmark generates. Since ACilk-5 saves the overhead of executing the memory fence whenever the victim 
accesses its deque, the more frequently a victim accesses its own deque, the more overhead ACilk-5 saves compared to Cilk-5.

Figure 5(b) shows the same performance comparison when executed on 16 cores. For most benchmarks, the execution time follows the same trend, where the normalized execution time is comparable between the serial execution versus the parallel execution; thus, the scalability of the two systems are comparable. The only obvious differences are in cholesky and lu, which do not scale as well under ACilk-5.

In the software implementation, the scalability of a benchmark under ACilk-5 is correlated with the ability to amortizes the overhead for sending / handling signals against successful steals. While the analysis of the work-stealing algorithm (referred as the "workfirst" principle [11]) dictates that one should put the scheduling overhead onto the steal path (thief's path) instead of onto the work path (victim's path), one must be able to amortize the overhead against successful steals in order to obtain good performance. In all benchmarks besides cholesky and lu, at least $90 \%$ of the signals sent by the thieves realize to successful steals, while in the case of cholesky, the signals-to-successful-steals ratio is only $53.6 \%$, and in the case of $l u$, the ratio is only $72.8 \%$. This means that high percentage of the signaling overhead in these two benchmarks cannot be accounted towards successful steals, and thus the performance suffers. With hardware support, the overhead in such case would be similar to a cache miss for the victim and an ordinary memory fence for the thief. Therefore we believe that the hardware mechanism would scale better even for these applications.

\section{Evaluation Using ARW Lock}

We evaluate the effect of location-based memory fences by comparing the read throughput of a microbenchmark using the ARW lock to the read throughput using its symmetric counterpart: the same design but uses the original symmetric Dekker protocol instead of the asymmetric Dekker protocol, henceforth referred as the $\boldsymbol{S} \boldsymbol{R} \boldsymbol{W}$ lock. Each thread performs read operations most of the time, and only occasionally it performs a write. In the tests, the threads read from and write to an array with 4 elements. The read-to-write ratio is an input parameter to the microbenchmark: assuming the ratio is $N: 1$, and there are $P$ threads executing, then for every $N / P$ reads, a thread performs a write. With each configuration, we run the microbenchmark for 10 seconds, calculate the read throughput, and compare the throughput using the ARW lock against the throughput using the SRW lock.

Figure 6(a) shows the throughput comparison between the ARW lock and the SRW lock. In the software prototype of location-based memory fences, since a request for serialization translates to a signal, the writer ends up signaling a list of readers and waiting for their responses one by one, which becomes a serializing bottleneck. This is particularly inefficient when the thread counts is high, and the read-to-write ratio is low.

We speculate that the lack of scalability is again due to the overhead of sending signals in the software implementation. To confirm this, we devised an ARW lock that implements a waiting heuristic: when a writer wants to write, instead of sending signals to the readers immediately, it first indicates intent to write and spin-waits to see if any reader responds, acknowledging the writer's intent to write. Only after spin-waiting for a while, the writer sends signals to readers who have not acknowledged. We refer to the ARW lock with this heuristic as the $\boldsymbol{A R} \boldsymbol{W}+$ lock.

Figure 6(b) shows the throughput comparison between the ARW+ lock and the SRW lock. A value above 1 means that the ARW+ lock performs better. There are two main trends to notice. First, as the number of threads increases, the ARW+ locks consis-

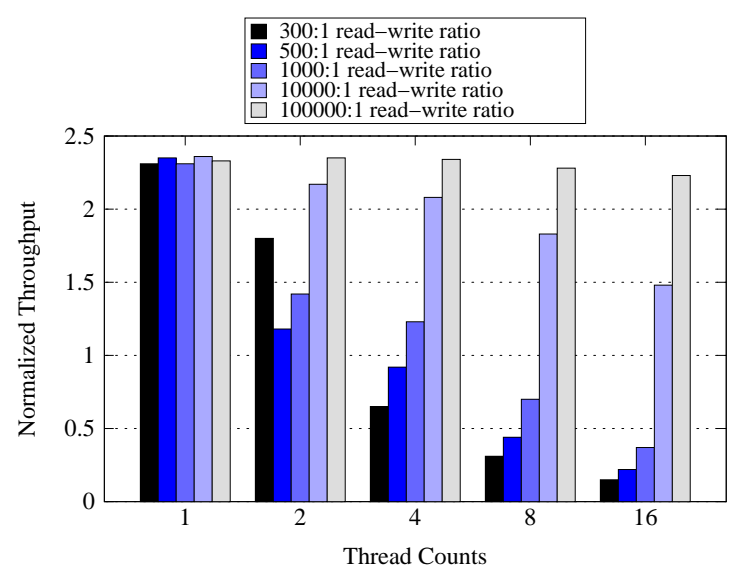

(a)

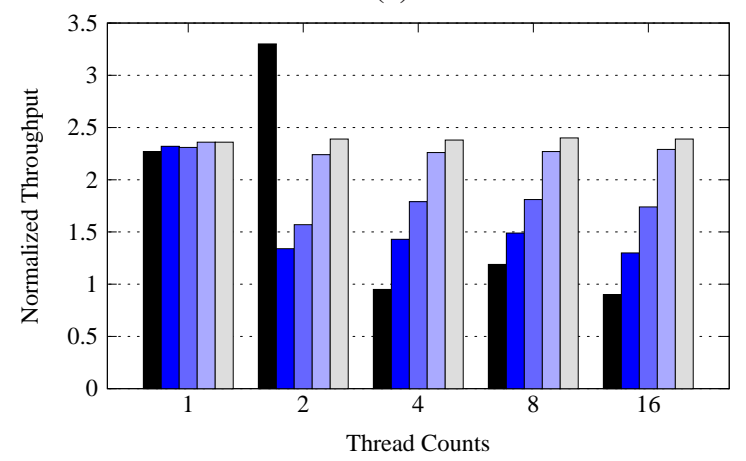

(b)

Figure 6: (a) The relative read throughput of execution using the ARW lock compared to that using the SRW lock. (b) The relative throughput of execution using the ARW+ lock (i.e., the ARW lock with the waiting heuristics) compared to that using the SRW lock. Since we are comparing the relative throughput, a value above 1 means that the ARW lock / ARW+ lock performs better; a value below 1 means that the SRW lock performs better. Each value is calculated by normalizing the read throughput from the execution using the ARW lock by that using the SRW lock.

tently have higher throughput compared to the SRW locks for each specific read/write ratio, except for the $300: 1$ ratio. Second, as the ratio between read and writes increases for a given thread count, the ARW+ consistently outperforms SRW, with one noticeable outliner: the data point for ratio of $300: 1$ with two threads. This can be explained by the fact that when there are only two threads, the writer end up receiving the acknowledgment most of the times and does not need to send signals. While the heuristic seems to work well in the microbenchmarks, if the reader does not acquire/release locks frequently in practice, the waiting heuristic would not help as much, since a thread would only get a chance to check for pending intent during lock acquire and release. With that in mind, the results inspire some confidence that ARW should exhibit good performance when implemented with hardware support for locationbased memory fences.

\section{RELATED WORK}

Our work is closely related to studies performed on biased locks and asymmetric synchronization, so we focus on these in the section. Several researchers studied this area, mainly in the context of improving performance for Java locks.

[23] describes a fast biased lock algorithm, which allows the primary thread to avoid executing memory fences, until a secondary thread attempts to enter the critical section. In which case, the sec- 
ondary thread must wait for the primary thread to grant access in order to continue execution. While this request and grant protocol is performed via shared variables and therefore fairly efficient, this implementation can potentially deadlock if the biased lock is nested within another lock (or any resource that can block).

The studies in [7] and [21] describe similar biased lock implementations, where the owner of the lock is on the fast path for accessing the lock, and other threads need to revoke it and compete for ownership, and the lock ownership may transfer. Both algorithms use the "collocation" trick, where the status field and the lock field are allocated on the same word. They first write to one field and then the whole word is read. The correctness of the algorithm depends on the fact that hardware typically does not reorder read before older write when the addresses overlap. This collocation trick, while interesting, is not guaranteed to be safe, and on systems which this trick works correctly, the collocation always forces a memory fence to be issued regardless of whether there is contention [8].

Serialization using signal and notify was proposed in [6], as well as other more heavy-weight serialization mechanisms. Their work focus on software means to cause serialization in another thread, while decreasing synchronization overhead on the primary thread in applications that exhibit asymmetric synchronization patterns.

Finally, in [20], Lin et al. propose a hardware mechanism for conditional memory fences, whose aim is also to reduce the overhead of memory fences when synchronization is unnecessary. In [20], however, the assumption is that the compiler would automatically insert memory fences in order to enforce sequential consistency everywhere, and there may be multiple outstanding memory fences for a given thread at a given moment. Thus, their hardware mechanism is much more heavyweight compared to ours, so as to handle multiple outstanding fences at a given moment. Our mechanism is designed for applications that are hand-tuned with manually inserted fences, and we aim to provide a lightweight solution which does not handle multiple outstanding fences.

\section{CONCLUSION}

In this work, we propose location-based memory fences, which aim to reduce the overhead incurred by memory fences in parallel algorithms. Location-based memory fences are particularly well-suited for algorithms that exhibit asymmetric synchronization patterns. We describe a hardware mechanism to support locationbased memory fences, proved its correctness and evaluate the feasibility of the fences using a software prototype. Our evaluation with the software prototype inspires confidence that the suggested LE/ST mechanism for supporting location-based memory fences in hardware is worth considering.

Finally, location-based memory fences lend itself to a different way of viewing programs compared to the traditional PC-based memory fences. It would be interesting to investigate what other algorithms can benefit from location-based memory fences, as well as other mechanisms that exploit the location-based model.

\section{ACKNOWLEDGMENTS}

We like to thank Joel Emer of Intel Corporation and MIT, David Dice of Oracle Labs, and William Hasenplaugh, Charles Leiserson, Jim Sukha, and other members of the SuperTech Group at MIT CSAIL for helpful discussions.

\section{REFERENCES}

[1] Advanced Micro Devices. AMD64 Architecture Programmer's Manual Volume 2: System Programming, June 2010.

[2] Nimar S. Arora, Robert D. Blumofe, and C. Greg Plaxton. Thread scheduling for multiprogrammed multiprocessors. In SPAA '98, pages 119-129, June 1998.

[3] Robert D. Blumofe, Christopher F. Joerg, Bradley C. Kuszmaul, Charles E. Leiserson, Keith H. Randall, and Yuli Zhou. Cilk: An efficient multithreaded runtime system. In $P P O P P$ ' 05 , pages 207-216, July 1995.

[4] Robert D. Blumofe and Charles E. Leiserson. Scheduling multithreaded computations by work stealing. JACM, 46(5):720-748, September 1999.

[5] Robert D. Blumofe and Dionisios Papadopoulos. Hood: A user-level threads library for multiprogrammed multiprocessors. Technical Report, University of Texas at Austin, 1999.

[6] Dave Dice, Hui Huang, and Mingyao Yang. Asymmetric dekker synchronization. Technical report, Sun Microsystems Inc., July 2001.

[7] Dave Dice, Mark Moir, and William Scherer III. Quickly reacquirable locks. Technical report, Sun Microsystems Inc., 2003.

[8] David Dice. David Dice's Weblog: http://blogs.sun.com/dave/ entry/biased_locking_in_hotspot\#comments, 2006.

[9] E. W. Dijkstra. Solution of a problem in concurrent programming control. Communications of the ACM, 8(9):569, September 1965.

[10] E. W. Dijkstra. Co-operating sequential processes. In Programming Languages. 1968.

[11] Matteo Frigo, Charles E. Leiserson, and Keith H. Randall. The implementation of the Cilk-5 multithreaded language. In PLDI '98, pages 212-223, 1998.

[12] Robert H. Halstead, Jr. Multilisp: A language for concurrent symbolic computation. ACM TOPLAS, 7(4):501-538, October 1985.

[13] John L. Hennessy and David A. Patterson. Computer Architecture: a Quantitative Approach. Morgan Kaufmann, San Francisco, CA, fourth edition, 2007.

[14] Intel Corporation. A Formal Specification of Intel Itanium Processor Family Memory Ordering, October 2011.

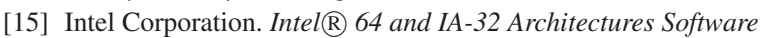
Developer's Manual Volume 3A: System Programming Guide, Part 1, January 2011.

[16] Kiyokuni Kawachiya, Akira Koseki, and Tamiya Onodera. Lock reservation: Java locks can mostly do without atomic operations. In OOPSLA '02, pages 130-141, 2002.

[17] David A. Kranz, Robert H. Halstead, Jr., and Eric Mohr. Mul-T: A high-performance parallel Lisp. In PLDI '89, pages 81-90, June 1989.

[18] Leslie Lamport. A new solution of dijkstra's concurrent programming problem. Communications of the ACM, 17(8):453-455, 1974.

[19] Leslie Lamport. How to make a multiprocessor computer that correctly executes multiprocess programs. IEEE Transactions on Computers, C-28(9):690-691, September 1979.

[20] Changhui Lin, Vijay Nagarajan, and Rajiv Gupta. Efficient sequential consistency using conditional fences. In $P A C T$ ' 10 , pages 295-306, 2010.

[21] Tamiya Onodera, Kiyokuni Kawachiya, and Akira Koseki. Lock reservation for java reconsidered. In ECOOP '04, pages 559-583, 2004.

[22] G. L. Peterson. Myths about the mutual exclusion problem. Information Processing Letters, 12(3):115-116, June 1981.

[23] Nalini Vasudevan, Kedar S. Namjoshi, and Stephen A. Edwards. Simple and fast biased locks. In PACT '10, pages 65-74, 2010.

[24] David L. Weaver and Tom Germond, editors. The SPARC Architecture Manual, Version 9. PTR Prentice Hall, 1994. 